Présentement, de nombreuses réflexions ont lieu concernant une recherche concomitante à I'occasion de l'introduction prochaine de SwissDRG. Alertés par certaines dérives observées dans les pays connaissant un tel système, les spécialistes de l'éthique se sont emparés du sujet et ont procédé à un état des lieux dans leur perspective. L'article ci-dessous fait état du résultat de ces réflexions: il va dans le même sens que le concept de recherche concomitante préconisé par la FMH, tout en le complétant par des réflexions éthiques spécifiques.

Lorsqu'il s'agira de mettre en œuvre une telle recherche, il sera nécessaire d'établir des priorités dans la définition des indicateurs pour lesquels des données seront récoltées, en mettant en balance les moyens financiers disponibles avec la masse d'informations souhaitable. Ce faisant, il serait inadmissible de ne prendre en compte que les paramètres économiques: les problèmes éthiques doivent être considérés à leur juste valeur et, par conséquent, figurer au premier plan des préoccupations de la recherche concomitante.

Dr Pierre-François Cuénoud, Membre du Comité central, responsable du domaine SwissDRG

\title{
Kriterien für die DRG-Begleitforschung aus ethischer Perspektive
}

Es besteht weithin Konsens, dass Effizienz im Gesundheitswesen wünschbar ist. Dabei stellt sich jedoch die Frage, wie ein Optimum an Effizienz ohne wesentliche Einbussen hinsichtlich eines fairen Zugangs zu einem qualitativ hochstehenden Gesundheitswesen erreicht werden kann. Wenn der Status quo sowie Veränderungen - wie derzeit die landesweite Einführung der DRGs - empirisch fundiert evaluiert werden sollen, ist eine gesundheitspolitische Verständigung über relevante Kriterien unumgänglich.

Verina Wild, Eliane Pfister, Nikola Biller-Andorno

1 Fleck L. DRGs: justice and the invisible rationing of health care resources. J Med Philos. 1987;12(2): 165-196.

2 Veatch RM. DRGs and the ethical reallocation of resources. Hastings Center Report. 1986;16(3):32-40.

Interessenbindungen: keine

Korrespondenz:

Prof. Dr. med. Dr. phil.

Nikola Biller-Andorno Institut für

Biomedizinische Ethik

Universität Zürich

Zollikerstrasse 115

CH-8008 Zürich

Tel. 0446348381

Fax 0446348389

biller-andorno@ethik.uzh.ch

\section{Zusammenfassung}

Das DRG-System zielt auf mehr Effizienz in der Spitallandschaft, in der Organisation individueller Spitäler und im direkten Umgang mit Patienten. Begleitforschung ist in ihrer Notwendigkeit weitgehend unumstritten, wird aber inhaltlich vielfach auf Qualitätssicherung anhand der üblichen Indikatoren und auf ökonomische Konsequenzen beschränkt. Aus ethischer Perspektive geht es jedoch nicht nur um Kostenaufwand bzw. -ersparnis einerseits und um medizinische Qualitätseinbussen andererseits.

Vielmehr geht es aus unserer ethischen Perspektive auch um feiner nuancierte Fragen des fairen $\mathrm{Zu}$ gangs zu einer hochqualitativen Versorgung, die u. a. auch psychosoziale Faktoren in der Patientenversorgung oder die Arbeitszufriedenheit von Ärzten und Pflegenden berücksichtigen und die gesamte Versorgungskette in den Blick nehmen.

Die bevorstehende, nicht unumstrittene schweizweite Einführung der DRGs bietet eine gute Chance, Begleitforschung im Gesundheitswesen zu institutionalisieren und sich über Kriterien zu verständigen, die nicht nur eine medizinische und ökonomische, sondern zugleich auch eine ethische Bewertung möglich machen. Der Beitrag stellt Elemente eines entsprechenden Kriterienkatalogs zur Diskussion.

\section{DRGs: Grundlagen für die ethische Begleitforschung}

Die Einführung der DRGs zur Abrechnung von Medicare-Leistungen hat in den USA bereits in den 1980er Jahren zu ethischen Debatten geführt [1-3]; auch die Einführung in Deutschland im Jahre 2003 wurde mit Blick auf ihre ethischen Implikationen diskutiert $[4,5]$. Auch wenn die konkreten Auswirkungen der DRGs von den spezifischen Gegebenheiten im jeweiligen Gesundheitswesen abhängen, so importieren sie doch prinzipiell einen Interessenskonflikt in die alltägliche ärztliche Arbeit - auf der einen Seite die bestmögliche Behandlung des Patienten, auf der anderen die Erlössteigerung oder zumindest das Vermeiden finanzieller Einbussen zum Wohle der eigenen Institution.

Ökonomische Anreize haben wohl seit jeher das ärztliche Handeln begleitet. Doch besteht ein qualitativer Unterschied zwischen einem Anreiz, der auf Gewinnsteigerung zielt (etwa durch überzogene Liegezeiten oder überflüssige Interventionen in Systemen mit Tagessätzen bzw. mit Fee-for-service-Prinzip), so dass derjenige, der ihm nachgibt, sich in moralischen Rechtfertigungsnöten findet, und einem Anreiz - seien es nun die DRGs oder andere Managed-Care-Elemente -, der sich durch das Gebot der Effizienzsteigerung bzw. der Kostendämpfung moralisch legitimiert, 
3 Donnelley S. Cost containment, DRGs and the ethics of health care. Introduction. Hastings Center Report. 1989;19(1):5 .

4 Lenk C, Biller-Andorno N, Alt-Epping B, Anders M, Wiesemann $C$. Ethik und Fallpauschalen. Welche Veränderungen in der Patientenversorgung sind zu erwarten? Dtsch Med Wochenschr. 2005;130(27):1653-55.

5 Wehkamp KH. DRGs als medizinethische Herausforderung. Dtsch Med Wochenschr. 2002;127:395-398.

6 Wild V, Pfister E, BillerAndorno N. DRG: Ethik vs. Ökonomie? SAMW-Bulletin. 2009:01:1-5.

Die Entwicklung eines umfassenden Kriterienkatalogs muss auf interdisziplinärem Weg entstehen; wir präsentieren hier Elemente aus unserer Fachperspektive.

Unter den 67 angeschriebenen Expertinnen und Experten befanden sich Vertreter der Ärzteschaft (13/8), Politik (3/1), Spitalmanagement $(5 / 3)$, Praxis $(11 / 5)$, Krankenkassen $(1 / 0)$ Patientenvertreter $(2 / 1)$ Behindertenorganisationen (1/1), Pflege (6/5), Medizinethik (7/3), SwissDRG AG

$(8 / 2)$, Ökonomie $(5 / 2)$, Begleitforschung (4/3) und Qualitätssicherung (1/0). Die Zahlen in Klammern bezeichnen die Anzahl Aussendungen und die Anzahl Antworten pro Expertengruppe.

Methodische Details sind im Referat von Prof. Nikola Biller-Andorno anlässlich des NEK-Symposiums vom 10.6.2009 zu finden, abrufbar unter: www.samw. ch/de/Projekte/DiagnosisRelated-Groups.html

4 Am Workshop vom 11.6.2009 in Zürich haben 12 Expertinnen und Experten teilgenommen.

Sitzung in Bern vom 26.6.2009.

Rückmeldungen aus de Leserschaft zu den im vorliegenden Beitrag dargestellten Elementen sind mit Blick auf eine Optimierung des Modells sehr willkommen. so dass sich nun derjenige, der sich dem Anreiz widersetzt, moralisch rechtfertigen muss.

Es ist diese Spannung zwischen Patientenwohl und betriebswirtschaftlicher Effizienz, die eine Begleitforschung auch aus ethischer Perspektive als besonders geboten erscheinen lässt. Dabei ist klar, dass es nicht um die Frage gehen kann, ob DRGs als «gut» oder «böse» zu beurteilen sind, sondern um ein Abwägen von Nutzen und Kosten (sowohl monetär als auch anderweitig) sowie um einen Vergleich des Nutzen-Kosten-Profils mit dem anderer Vergütungssysteme. Auf dieser Basis kann die Entscheidung für die DRGs empirisch und argumentativ fundiert bestätigt, im Detail modifiziert oder auch revidiert werden.

Voraussetzung für eine solche Evaluation ist jedoch die Festlegung, wie sich der Nutzen einerseits und die Kosten andererseits bemessen lassen sollen. Woran wird man feststellen können, ob die Einführung der DRGs ein Erfolg war? Und wann wäre der Punkt gekommen, an dem man die Kosten als zu schwerwiegend betrachten würde? Mit welchen Indikatoren wollen wir den fairen Zugang zu einer qualitativ hochstehenden Medizin, wie er in der Schweiz allgemein angestrebt wird, erfassen?

Das vom Käthe-Zingg-Schwichtenberg-Fonds der Schweizerischen Akademie der Medizinischen Wissenschaften (SAMW) geförderte und vom Institut für Biomedizinische Ethik (IBME) der Universität Zürich durchgeführte Projekt «Fallpauschalen in Schweizer Spitälern: Grundlagen für die ethische Begleitforschung» hat sich diesen Fragen zugewandt und auf Basis einer umfassenden Literaturrecherche sowie von Experteninput u.a. aus den Bereichen Medizin, Pflege, Spitalmanagement, Medizinethik, Ökonomie und Gesundheitspolitik einen Vorschlag für Elemente eines entsprechenden Kriterienkatalogs als Diskussionsgrundlage erarbeitet. ${ }^{1}$

\section{Die Erarbeitung ethisch relevanter Kriterien - ein mehrstufiger Prozess}

Ziel des Projektes war die schrittweise Erarbeitung der inhaltlichen und methodischen Grundlagen einer DRG-Begleitforschung aus ethischer Perspektive. Hierfür wurde zunächst die schweizerische wie auch die internationale Literatur zu den ethischen Implikationen fallpauschalierter Vergütungssysteme im stationären Bereich rezipiert und mit Blick auf ihre Relevanz für den Kontext des Schweizer Gesundheitswesens analysiert [6]. Die aus der Literatur gewonnenen Einsichten wurden ergänzt durch eine aktive Auseinandersetzung mit der Fragestellung im Rahmen eines am IBME durchgeführten Forschungskolloquiums, erste explorative (Experten-)Gespräche im Schweizer Raum sowie die Auswertung von Tagungsbesuchen und Vortragsdiskussionen in der Schweiz.

Ein erster Entwurf von Kriterienclustern wurde im Rahmen einer kleinen Delphi-Studie verschiedenen Stakeholdern im Schweizer Gesundheitswesen zur Kenntnis und Bearbeitung gegeben; ${ }^{2}$ auf diese Weise konnte ein Konsens eruiert werden bezüglich der

\section{Critères pour la recherche concomitante aux DRG, sous une perspective éthique}

Le système des DRG a pour objectif une meilleure efficacité dans l'univers de l'hôpital, dans l'organisation des cliniques et dans les contacts directs avec les patients. Si quasiment personne ne conteste la nécessité d'une recherche concomitante, on en restreint toutefois souvent le contenu à l'assurance qualité, sur la base des indicateurs habituels, et aux conséquences économiques. Toutefois, du point de vue de l'éthique, il ne s'agit pas uniquement de dépenses ou d'économies d'une part et de pertes de qualité des soins d'autre part. Il s'agit bien plus, dans la perspective éthique dans laquelle nous nous situons, de questions subtiles d'accès équitable à des soins de grande qualité, qui prennent également en compte des facteurs psychosociaux pour le traitement des patients ou la satisfaction au travail des médecins et du personnel soignant et considèrent l'intégralité du système de soins.

Le lancement des DRG à l'échelon national est prévu et personne ne le conteste. II offre une opportunité idéale d'institutionnaliser la recherche concomitante dans le secteur de la santé et de clarifier des critères qui permettent une évaluation non seulement médicale et économique mais également éthique. L'article ouvre le débat sur des éléments d'un catalogue de critères dans ce domaine.

Frage, welche ethischen Aspekte bei der Einführung der SwissDRGs bedeutsam sind und wie diese angemessen berücksichtigt werden können. ${ }^{3}$ Diese Ergebnisse wurden im Rahmen eines Workshops am IBME konsolidiert. ${ }^{4}$

Nach der Entwicklung einer graphischen Darstellungsform der zentralen Resultate (vgl. Abbildungen 1-3) wurden schliesslich die synthetisierten Ergebnisse der Zentralen Ethikkommission der SAMW präsentiert. ${ }^{5}$ Eine finale Version mit weiteren Ausführungen soll schliesslich den an der Umsetzung der DRGs massgeblich beteiligten Instanzen für die Umsetzung der DRGs, insbesondere dem Bundesamt für Gesundheit, der FMH und der SwissDRG AG, zur Kenntnis gegeben werden. ${ }^{6}$

\section{Elemente für einen Kriterienkatalog}

Die Einführung der DRGs hat auf wesentliche Bereiche der Gesundheitsversorgung in der Schweiz Ein- 


\section{Abbildung 1}

Kriteriencluster A - Patientenversorgung und Angehörige.

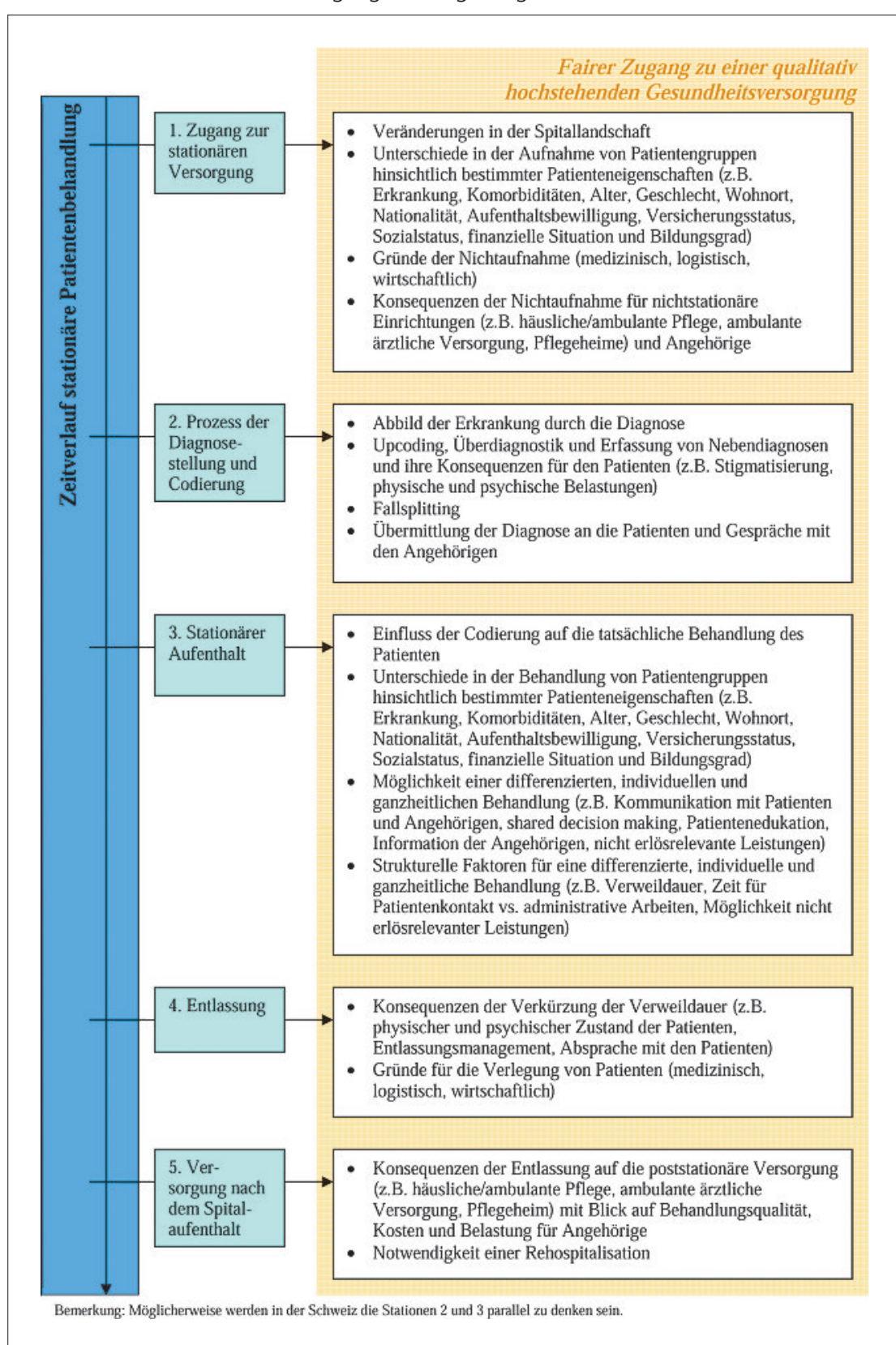

fluss. In unserer Studie haben wir drei Bereiche als besonders wichtig für eine ethische Begleitforschung identifiziert. An erster Stelle ist hier die Patientenversorgung zu nennen, zu der wir auch das beteiligte soziale Umfeld (z.B. Angehörige) zählen. Des weiteren haben DRGs Einfluss auf die «Leistungserbringer», in erster Linie Ärzte und Pflegende im Spital. Sowohl Patienten als auch Mitarbeitende sind Akteure innerhalb institutioneller und gesellschaftlicher Rahmenbedingungen, die wir als dritten Bereich identifizieren, der durch die Einführung der DRG beeinflusst wird und aus ethischer Sicht von Bedeutung ist.

Die von uns erarbeiteten ethisch relevanten Kriterien für die DRG-Begleitforschung sind daher in drei
Kriteriencluster aufgeteilt: A. Patientenversorgung und Angehörige, B. Professionelles Selbstverständnis und C. Institutionelle und gesellschaftliche Bedingungen.

Die folgenden Kriterien sind keinesfalls als abschliessende «Liste» zu verstehen, sondern als eine Zusammenstellung, die kontinuierlich im Licht neuer Erkenntnisse ergänzt und modifiziert werden kann. Insgesamt sollte die Begleitforschung selbstreflexiv bleiben, um auch neu auftretende Problemfelder adäquat erfassen zu können.

\section{A. Patientenversorgung und Angehörige}

Die nebenstehende Abbildung 1 orientiert sich am chronologischen Verlauf einer Patientenbehandlung ab Aufnahme ins Spital bis zur Entlassung / poststationärer Verlauf. Links werden die zeitlich nacheinander folgenden Schritte eines Verlaufs von Aufnahme bis Entlassung / poststationärer Verlauf dargestellt und rechts die im Rahmen des Projekts identifizierten ethisch relevanten Kriterien, die es in einer DRG-Begleitforschung zu untersuchen gilt.

\section{B. Professionelles Selbstverständnis}

Der zweite zentrale Bestandteil der Gesundheitsversorgung sind die Gesundheitsleistungserbringer, die mit einem je Berufsgruppe eigenen professionellen Selbstverständnis im realen Arbeitsumfeld agieren. Das ärztliche und pflegerische Ethos stellt eine Art Anspruch an den eigenen Beruf und seine Ausübung dar, der sich mit der Wirklichkeit - den realen Arbeitsbedingungen - decken kann, aber nicht notwendigerweise muss. Neue Gesundheitsreformen, wie beispielsweise die Einführung des DRG-Systems, sollten deshalb bei ihrer Einführung auch daraufhin geprüft werden, ob sie einen Einfluss auf die Deckung von professionellem Ethos und realem Arbeitsumfeld haben. Eine mögliche Diskrepanz zwischen Anspruch und Wirklichkeit kann Konsequenzen nicht nur für die Berufsausübenden selber, sondern auch für die Patienten haben (siehe Abbildung 2).

\section{Institutionelle und gesellschaftliche Bedingungen}

Die schematische Abbildung 3 verdeutlicht, dass die Cluster A und B nur im Kontext von Cluster C den institutionellen und gesellschaftlichen Rahmenbedingungen als drittem zentralen Bereich - exploriert werden können, und dass das professionelle Selbstverständnis und die Qualität und Möglichkeiten der Patientenversorgung einander durch Interaktion gegenseitig beeinflussen

\section{Methodische Aspekte}

Das Studiendesign einer ethischen DRG-Begleitforschung, welche die genannten ethisch relevanten Kriterien (oder eine Auswahl davon) evaluiert, sollte die folgenden methodischen Merkmale aufweisen:

- Die Evaluation sollte sich nicht auf quantitative (z. B. Fragebogen) Elemente beschränken, sondern 
auch qualitative Elemente (z.B. Interviews) enthalten.

- Die Erarbeitung der Items sollte in Fokusgruppen mit Beteiligung der Zielgruppen erfolgen.

- Die Evaluation sollte als Längs- (vergleichend über einen längeren Zeitraum) und Querschnitt-

\section{Abbildung 2}

Kriteriencluster B - Professionelles Selbstverständnis.

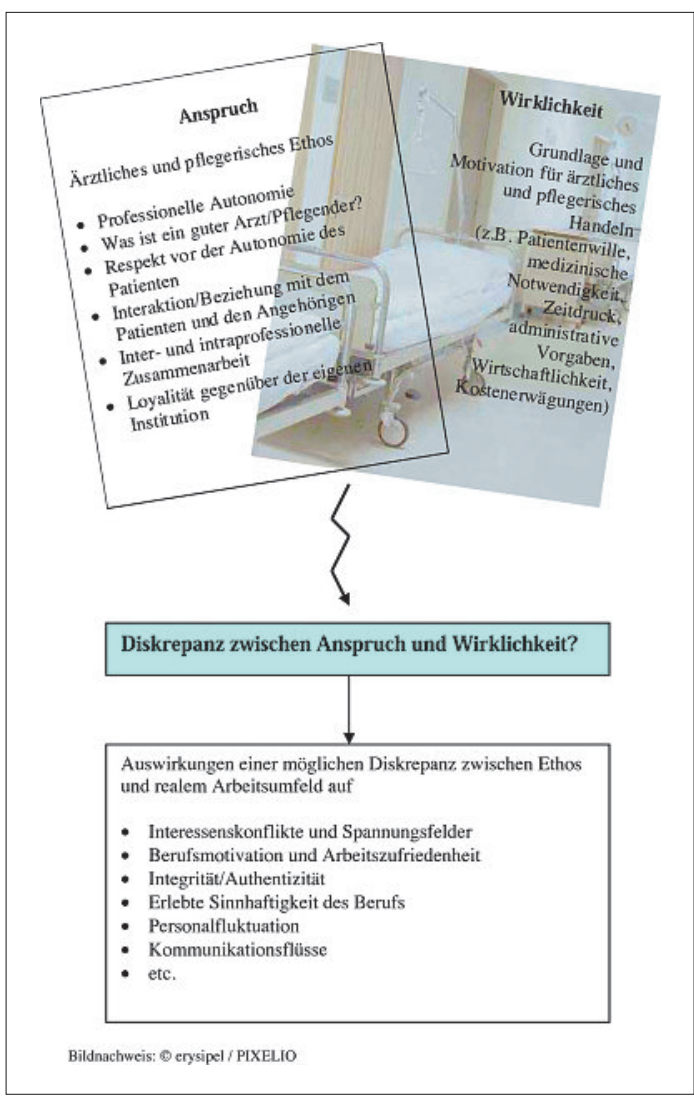
RL. Benchmarks of fairness for health care reform. Oxford

University Press; 1996.

\section{Abbildung 3}

Kriteriencluster C - Institutionelle und gesellschaftliche Bedingungen.

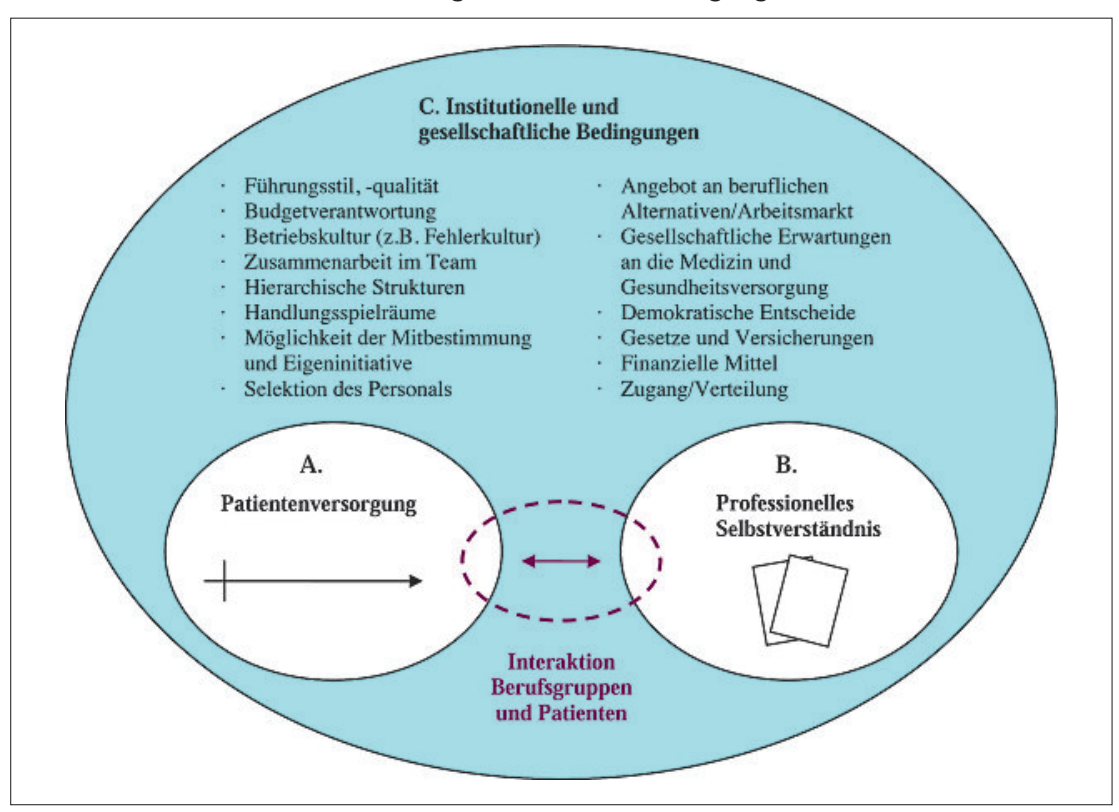

studie (Erhebung zu einem Zeitpunkt) durchgeführt werden.

- Die Evaluation sollte weit über den Einführungszeitraum hinaus fortgesetzt werden, da einige Effekte erst im späteren Verlauf erwartet werden können.

- Die Ergebnisse der Evaluation müssen fachkundig ausgewertet, mit ethischer Expertise beurteilt und dem öffentlichen Diskurs zugänglich gemacht werden.

Ein Studiendesign, das für die Erhebung aussagefähiger Daten konzipiert wird, wird aber auch mit nicht zu vernachlässigenden methodischen Schwierigkeiten konfrontiert. Um der methodischen Komplexität gerecht zu werden, ist die Zusammenarbeit verschiedener Disziplinen - insbesondere der Medizin, der Pflege, der Ethik, der Sozial- sowie der Rechtswissenschaften und der Ökonomie - unabdingbar.

\section{Ausblick}

Eine Begleitforschung, die sich nicht auf einige grobe medizinische oder ökonomische Indikatoren beschränkt, sondern auch ethisch relevante Kriterien einbezieht, ist kein triviales Unternehmen. Sie ist methodisch komplex, auf interdisziplinäre Kooperationen und auf entsprechende Ressourcen angewiesen. Dafür bietet sie die Chance, Veränderungen im Gesundheitswesen, die sich nachhaltig auf die gesundheitliche und psychosoziale Situation vieler Individuen auswirken können, angemessen zu erfassen.

Auf diese Weise wird zugleich der gesundheitspolitische und gesellschaftliche Diskurs zur Mittelallokation im Gesundheitswesen entscheidend bereichert: Nicht nur wird transparent, an welchen Kriterien wir ein faires und gutes Gesundheitswesen festmachen wollen, sondern es wird auch deutlich, welche Kosten die Einführung ökonomischer Steuerungsinstrumente mit sich bringen kann.

Fragen wie «Erkaufen wir uns die Effizienzsteigerung im stationären Setting um den Preis einer Reduktion an ärztlicher und pflegerischer Zuwendung?» oder «Werden Kosten aus dem stationären in den nachstationären - ambulanten oder auch privaten Bereich verlagert?» sollten nicht nur im Vorfeld der DRG-Einführung antizipativ thematisiert und dann ad acta gelegt werden. Vielmehr verdienen diese Fragen offene, empirisch fundierte Antworten, ganz im Sinne der accountability, die Norman Daniels als eine der benchmarks of fairness [7] definiert hat. Die nachhaltige Legitimierung von Reformen im Gesundheitswesen würde auf diesem Wege entscheidend befördert.

\section{Danksagung}

Die Autorinnen danken allen Expertinnen und Experten, die sich die Zeit genommen haben, sich mit den Projektergebnissen in ihren verschiedenen Stadien auseinanderzusetzen. 